\title{
Informes
}

\section{Homenaje a Alejandro Mina}

\section{Tribute to Alejandro Mina}

\section{Resumen}

El Colegio de México rindió un Homenaje a Alejandro Mina, destacado demógrafo y formador de muchas generaciones de estudiosos de la población. El acto póstumo se llevó a cabo el 6 de febrero de 2019 en la Sala Alfonso Reyes de las instalaciones de dicha institución.

A continuación, reproducimos las intervenciones de los oradores en el orden de su presentación: Manuel Ordorica, profesor-investigador del Centro de Estudios Demográficos y Urbanos (CEDUA) de El Colegio de México; Carlos Javier Echarri Cánovas, profesor-investigador del CEDUA, El Colegio de México y secretario general del Consejo Nacional de Población; Silvia E. Giorguli, presidenta de El Colegio de México; y Mauricio Rodríguez Abreu, profesor de la Universidad de las Américas, Puebla. El acto fue moderado por Jaime Sobrino, director del CEDUA, El Colegio de México.

\begin{abstract}
El Colegio de México paid tribute to Alejandro Mina, an outstanding demographer and teacher of many generations of population students. The posthumous act was held on February 6, 2019 in the Alfonso Reyes Hall of this institution.

The addresses of the speakers are reproduced below in order of appearance: Manuel Ordorica, research fellow at the Center for Demographic and Urban Studies (CEDUA) of El Colegio de México; Carlos Javier Echarri Cánovas, research fellow at CEDUA, El Colegio de México and general secretary of the National Population Council; Silvia E. Giorguli, president of El Colegio de México; and Mauricio Rodríguez Abreu, professor at the University of the Americas, Puebla. The act was moderated by Jaime Sobrino, director of CEDUA, El Colegio de México.
\end{abstract}




\section{Palabras de Manuel Ordorica Mellado*}

En primer lugar, quiero agradecer a Silvia Giorguli, presidenta de El Colegio de México, y a Luis Jaime Sobrino, director del CEDUA, la invitación a decir unas palabras en este homenaje a mi amigo y colega Alejandro Mina.

\section{El primer encuentro}

La primera vez que nos vimos Alejandro Mina y yo no fue ni en la Facultad de Ciencias, ni en El Colegio de México; fue en el gimnasio de la SCOP (Secretaría de Comunicaciones y Obras Públicas). Él ahí jugaba básquetbol y yo fui a entrenar con el equipo mexicano de bádminton. Íbamos a jugar contra Canadá. Los basquetbolistas querían conocer ese deporte y se quedaron al entrenamiento. Por ahí andaba Alejandro, siendo él un jugador juvenil de básquetbol que estaba viendo los entrenamientos del equipo mexicano de bádminton, ahí estaba yo. Esto me lo comentó Alejandro.

La segunda ocasión que nos vimos, y ahí nos conocimos, fue en El Colegio de México. Él se encontraba en la puerta de uno de los salones de clase de este edificio. Yo llegaba a dar la clase de Matemáticas. Me preguntó: “¿Tú también vas a llevar el curso de Matemáticas?” Le dije: “No, yo soy el profesor, yo ya hice la maestría". Ya no dijo nada y nos reímos. Siempre fue muy respetuoso de mi clase. Curioso, pero él y yo no nos conocimos antes en la Facultad de Ciencias.

\section{Como alumno}

Fue un excelente estudiante con una muy buena formación matemática. Pero, sobre todo, siempre fue generoso. Siendo estudiante de la maestría apoyaba a los compañeros del grupo que no sabían matemáticas. Les daba clase de Análisis Demográfico de 7 a 8 de la mañana. Les repetía la clase que el profesor había dado unos días antes. Es importante reconocer que la suya fue una muy buena generación de estudiantes de Demografía y varios competían con él. Siempre identificaba a sus compañeros y compañeras por dichos o acciones que hacían cuando sufrían en un examen. Tenía a sus amigas y compañeras muy identificadas por sus palabras. Me comentaba que Guadalupe Salas decía: "Me quiero morir", y Luisa Benítez se subía a la paleta del

* El Colegio de México, A.C. Correo: mordori@colmex.mx 
pupitre y exclamaba "Me voy a suicidar". Siempre tuvo un gran sentido del humor y era muy querido por sus compañeros y compañeras.

\section{Como profesor}

Fue un excelente maestro. Yo describiría su método de enseñanza como la técnica del paso a paso; por cierto, un sistema muy didáctico. A los alumnos que no iban bien en el curso, los citaba en su cubículo para aclarar conceptos y ayudarlos a mejorar. A veces les dejaba un trabajo especial para elevar su nota. Cuando pensaba que algún estudiante debería tener una nueva oportunidad, le realizaba un examen especial o le dejaba ejercicios adicionales para mejorar su nivel. Se aseguraba que dominaran la materia. Siempre fue muy humano, positivo y riguroso.

Yo diría que fue un profesor muy observador de su grupo de estudiantes. Conocía su vida para ayudarlos. Sabía cuándo los alumnos y alumnas tenían un problema familiar o sentimental, una enfermedad, un accidente y más. Le tenían mucha confianza. Fue un tutor natural de los estudiantes, yo diría que hasta terapeuta de muchos de ellos. Los conocía muy bien. Identificaba con mucha puntería quiénes podían continuar en el programa de posgrado. Enseñaba para que todos sus alumnos entendieran la materia y sabía quiénes venían de áreas no matemáticas para darles un apoyo mayor. Sus cursos eran prácticos para que los estudiantes que venían de áreas no matemáticas pudieran entender primero la práctica y luego la teoría, para evitar la mecanización de conceptos, que es algo que ocurre con frecuencia en nuestro ambiente matemático.

\section{La vida en El Colegio de México}

Como investigador de El Colegio de México puedo decir que siempre fue muy institucional. Buen compañero, siempre buscaba conciliar las distintas opiniones, sobre todo cuando se acaloraban los ánimos de los colegas, lo que era bastante seguido, como suele ocurrir de manera natural en el ámbito académico. Fue coordinador del posgrado en Demografía, director de la revista y perteneció a todos los órganos colegiados del CEDUA. No le gustaba la política en el interior de la institución. Se aislaba de eso. Si bien es cierto que asistía a las reuniones nacionales de demógrafos, nunca quiso presidir la Somede, aunque lo hubiera hecho muy bien. No ambicionaba el poder y siempre fue muy ecuánime, sencillo y profesional. 
Apoyó a otras instituciones en sus programas de demografía. Fungió como profesor de Matemáticas y Análisis Demográfico en la maestría en Población de Flacso. Fue un investigador productivo, autor de 67 artículos de investigación y dos libros.

\section{Sus cursos y preferencias}

Alejandro Mina dio algunos cursos de Economía Matemática y muchos años de Demografía. Con el profesor Manuel Ángel Castillo impartió conjuntamente el curso de Migración por varios años. Juntos dimos el curso de Demografía Matemática a varias generaciones.

Yo creo que una de las materias que más lo entusiasmaba era la de Métodos Numéricos. Le gustaba evaluar y ajustar datos para darles coherencia y poder entender y explicar el comportamiento de los componentes demográficos; Gregory-Newton, Euler, Newton-Raphson, Sprague, el de 1/16, etcétera, estaban en muchos de sus textos. Entre otras cualidades, en los métodos numéricos primero evaluaba su calidad y luego los corregía. Algo que hoy se hace poco. Se piensa que las cifras son perfectas y se meten al modelo sin evaluación. Decíamos que si al modelo lo alimentas con basura, basura sale. Coincidíamos en que hay trabajos con miles y miles de cifras, como por ejemplo las tablas de mortalidad por edades individuales; tienen 2400 datos si tomamos en cuenta las ocho variables que hay, para hombres, para mujeres y para el total. ¿Analizamos todo con cuidado? De esos números, sólo se analizan unos cuantos. Considerábamos que teníamos que enseñar a los estudiantes a leer los números principales y a interpretar y demostrar las fórmulas. En pocas palabras, eliminar la mecanización. El gusto por los métodos numéricos habla de su personalidad. Corregir los datos tiene un símil en la vida, ayudar al mejor comportamiento ético y profesional de las personas. Su principio fue siempre evaluar la calidad del dato para luego incorporarlo al modelo.

\section{En la UNAM}

Alejandro Mina apoyó a muchos estudiantes de Actuaría y de Matemáticas de la UNAM para que terminaran su tesis, y dirigió decenas de ellas. Para ser más exactos, dirigió 165 tesis de licenciatura y 38 de posgrado. Siempre de muy buena gana y tenacidad, los impulsaba a terminar su licenciatura. 
Comentábamos él y yo la suerte de vivir en estos dos decenios del siglo $\mathrm{XXI}$, con pocos cambios en temas trascendentales en la natalidad y en la mortalidad, y en algunos aspectos socioeconómicos. Cada vez hay más pobres, más desigualdad, la esperanza de vida al nacer se estancó en 75 años $\mathrm{y}$, por lo que se ve según las proyecciones de población, seguirá sin muchos cambios durante este sexenio. Esperemos que no sea así. Decíamos que las nuevas generaciones tendrán mucho trabajo para remontar esa situación.

Comentábamos también la suerte que habíamos tenido en nuestra vida por ver grandes cambios: la esperanza de vida al nacer había aumentado de 50 a 75 años entre 1950 y 2000, mientras la tasa global de fecundidad descendió de 7 a 2 hijos por mujer, en promedio. Afortunados no sólo por ver los números, sino por observar en la vida cotidiana dichos cambios.

\section{En los últimos días}

Platicamos sobre la docencia y sobre los planes de pensiones. Nos daba mucha risa pensar que hay palabras que dejan perplejos y apantallan a los neófitos, como, por ejemplo: kernel, ergodicidad, estocástico, momentum, etc. La mayoría de las personas se quedan boquiabiertas con esas palabras y piensan que se trata de trabajos científicos de alto nivel de complejidad. Respecto a los planes de pensiones, comentábamos que muchas ideas no han cambiado mucho desde que Bismarck creó los sistemas de seguridad social.

En los últimos días antes de su fallecimiento me platicó de sus planes en el sabático y de su viaje futuro.

Para terminar, quiero mencionar que fue un gran amigo, que lo vamos a extrañar y que vamos a recordar sus intervenciones para buscar la mejor comunicación entre las personas y entre las generaciones de demógrafos.

Lo vamos a extrañar porque fue generoso con sus alumnos y compañeros.

Lo vamos a extrañar porque su forma de ser permitió ayudar a unir a la generación de profesores de mayor edad con los jóvenes. Decíamos que en El Colegio no habíamos atendido a tiempo la renovación de la planta académica. Modificamos la demografía del país, pero no la demografía de El Colegio, lo que apenas estamos viendo en el presente.

Lo vamos a extrañar porque impidió que tomáramos decisiones erróneas con los estudiantes.

Lo vamos a extrañar en los cursos de Análisis Demográfico por haber sido un excelente profesor.

Lo vamos a extrañar porque fue muy institucional buscando siempre colaborar con El Colegio. 
Lo vamos a extrañar porque se fue en un momento de gran productividad académica, sabiduría y madurez.

Lo van a extrañar sus familiares porque fue buen esposo, buen papá y buen abuelo.

Lo van a extrañar sus alumnos, a quienes siempre apoyó en las clases y en sus tesis.

En fin, lo vamos a extrañar y, más bien, ya lo estamos extrañando. 


\section{Palabras de Carlos Javier Echarri Cánovas*}

Comienzo por agradecer esta invitación a tan merecido homenaje. Lo que quiero compartir con ustedes es, más que de los temas de los que ya nos habló Manuel Ordorica, cuál fue mi experiencia con él desde el lado más personal.

Conocí a Alejandro Mina en la Facultad de Ciencias, por ahí del año 1983, cuando estaba yo joven, con cabello y flaco. Como ustedes saben, en la carrera de Actuaría hay un curso obligatorio de Demografía, en el que había dos opciones: entré a una clase de la primera opción y salí corriendo, entonces fui con Alejandro Mina. Él era muy buen profesor, muy claro, pero también con gran calidad humana. En alguna ocasión -pues está uno joven y pensando en otras cosas-, llegué a la clase y vi que todo el mundo guardó los libros y sacó el papel: ¡había examen y yo ni enterado! De la cara que puse, se acercó y le dije: "sabe, perdóname, pero si lo dijo, no lo oí o se me fue", y él, con la mayor tranquilidad del mundo, me dijo: "no te preocupes, yo sé que sabes", y ahí quedó el asunto.

Después, como vio mi interés por la demografía, me dijo "en el Centro Interamericano de Estudios de Seguridad Social van a dar un diplomado sobre temas de pensiones y seguridad social, eso te puede interesar, ¿por qué no vas?”. Fui y me inscribí. Ahí fue donde conocí más el mundo de la demografía y a los profesores que eran colegas de Alejandro, entre ellos a Manuel Ordorica y a Julieta Quilodrán, porque el curso lo daban profesores de El Colegio de México, quienes se habían juntado para dar este breve diplomado. También conocí a Catherine Menkes, a Elena Zúñiga y a otras personas. Entonces nació mi interés por la demografía.

Cuando llegó el momento de hacer la tesis, mi elección natural para que me la dirigiera fue Alejandro Mina. Yo fui la decimoctava persona a quien le dirigió la tesis; puede decirse que alcanzó la mayoría de edad como profesor conmigo, pero no se quedó ahí, porque finalmente fueron 165 personas a las que les dirigió la tesis de licenciatura. Podría alguien pensar que si a esa edad, en el año 1984, ya había dirigido 18 tesis, Alejandro era una fábrica de tesis. Pero no, él ponía atención a cada uno de sus dirigidos y nos orientaba sobre cuáles eran los problemas. Recuerdo, por ejemplo, que en aquellas épocas donde no había computadoras ni el acceso a la información que ahora tenemos, me canalizó al INEGI: "te voy a mandar con una persona para que te dé acceso a los datos". Ahí fue donde conocí a María de la

* El Colegio de México, A.C., Centro de Estudios Demográficos, Urbanos y Ambientales, y Consejo Nacional de Población. ORCID: https://orcid.org/0000-0001-5289-960X 
Paz López Barajas, que es egresada de aquí -y ahí inició también una relación de amistad y de trabajo que se mantiene hasta ahora-; cuando le dije que me mandaba Alejandro Mina, se me abrieron las puertas, me dieron toda la información para hacer una tesis sobre migración interna con los datos del Censo de 80 (desafortunadamente, tras el sismo de 1985 INEGI nunca sacó esa parte de la información debido a que se perdió junto con la computadora del instituto).

Además del seguimiento de los estudiantes, que siempre realizaba con mucho respeto, también se interesaba en la parte humana y en los problemas personales. Por azares de la vida, yo me quería recibir muy pronto para ser el actuario titulado más joven. Metí mis papeles a Asuntos Escolares de la Facultad de Ciencias, donde me respondieron que me faltaba un papel: mi certificado de nacionalidad. Pregunté dónde se hacía el trámite, lo saqué muy rápido, regresé, lo metí y no pasaba nada. Le conté a Alejandro, quien me dijo "ve y pregunta en Rectoría que está pasando". Fui, pregunté y me dijeron que yo no había metido ningún papel. Entonces Alejandro me dijo "lo que pasa es que quieren que le des dinero. No vayas a permitirlo", y yo tampoco iba a prestarme a la corrupción. Me peleé y como por arte de magia salió mi papel. Pero pude hacer mi examen hasta dos meses después de mi cumpleaños; entonces ya no fui el más joven en titularme, pero de todas maneras fui bastante joven como actuario.

Con esa experiencia, Alejandro me recomendó que hiciera la maestría en Demografía en El Colegio de México. Yo estaba buscando opciones fuera del país, pero él me dijo que la formación de El Colegio era muy buena y que se ajustaba a lo que yo quería. Entramos a un propedéutico, así como Luis Jaime Sobrino. Fuimos una promoción, digamos, especial y él entró al quite con nuestra promoción. Lo recordamos como un buen profesor que nos ayudó y nos guío.

Si bien Alejandro ya no me dirigió la tesis de maestría, sí incluí todos los temas de corrección de ajuste de información que él nos había enseñado de manera muy precisa. Con esto lo que quiero decir es que sus enseñanzas, yo creo que a todos los que fuimos sus alumnos, nos dejaron marcado el interés por la rigurosidad de los datos, por hacer bien las cosas, por el manejo de la información. Ése fue un aprendizaje no exclusivo para mí, sino para todos, incluyendo la preocupación de que la enseñanza fuera clara, incluso para aquellos compañeros que venían de otras formaciones, como de ciencias sociales u otras. Queríamos, tanto él como nosotros, que toda la promoción tuviera la misma formación.

Yo me fui a trabajar, hice el doctorado y luego regresé a El Colegio. Entonces Alejandro se convirtió, además de mí mentor y director de tesis de 
licenciatura, en colega de trabajo, en compañero en el Centro de Estudios Demográficos Urbanos y Ambientales. Aquí me tocó a mí coordinar la maestría en dos ocasiones y en otra el doctorado. De esas experiencias, quiero resaltar que Alejandro siempre tuvo una actitud propositiva y un ánimo permanente para resolver problemas. Su interés era definir qué es lo mejor para los estudiantes. Como en toda comunidad humana, siempre tenemos diversidad de opiniones y discusiones, pero él era un factor de unión, de solución, más que un factor de división. Así fue todo el tiempo.

Lo recordaremos siempre. Creo que nos vamos a pelear siempre si era nuestro o si era de la Facultad de Ciencias, pero en ambos lugares Alejandro mantuvo todo el tiempo esa disposición de ayudar.

Yo no conocí, como Manuel Ordorica, sus primeras etapas, pero sí estoy familiarizado con las historias de sus compañeros de promoción, de cómo los ayudaba, de todo lo que hacía por ellos. Si uno revisa en su currículum, que es impresionantemente largo, verá muchos nombres de personas que fueron sus alumnos, y que después destacaron tanto en la actuaría como en la demografía.

La última vez que me tocó compartir con él fue en la fiesta de fin de año. Estaba muy orgulloso de su familia. Eso nos muestra también cómo apreciaba el valor de la familia, de tener una actitud positiva, propositiva, de disfrutar la vida, de ser alguien amable con todos. Así era Alejandro Mina, así es como lo recuerdo. Estas son mis últimas percepciones de él, y así es como lo voy a mantener en mi mente y en mi corazón. 


\section{Palabras de Silvia E. Giorguli*}

\section{Alejandro Mina: gran profesor, ejemplo de entrega, amigo y excelente ser humano}

Agradezco a Luis Jaime Sobrino y al Centro de Estudios Demográficos, Urbanos y Ambientales la organización de este muy merecido y necesario homenaje a mi querido profesor Alejandro Mina.

Un saludo especial para Aída, Aleida chica y Aleida grande, a Alejandro $y$, en fin, a toda la familia de Alejandro Mina que nos acompaña. También a sus amigos y colegas de fuera de El Colegio de México.

Finalmente, saludos a la comunidad del Colmex, al gremio de demógrafos y a los miembros de las múltiples generaciones de actuarios, demógrafos y estudiantes de varios programas del Colmex y de la UNAM que fueron sus alumnos.

Para mí es un honor y un reto participar en este homenaje. No podía faltar porque soy una de las alumnas de Mina y porque tengo mucho que decir sobre cómo permeó mi visión: del oficio del demógrafo, de la docencia que hacemos en El Colegio, y de los balances entre la vida profesional -que puede ser apasionante- $\mathrm{y}$ la vida familiar, que es un motor de nuestro compromiso cotidiano.

Le agradezco y reconozco también su gran cariño y compromiso con esta institución, en la cual trabajó por casi cuarenta años.

\section{Alejandro Mina: un docente excepcional}

En palabras de sus alumnos:

Para mí, Alejandro fue el mejor profesor que he tenido. Atento, paciente, tolerante. Nunca he visto a alguien que enseñe matemáticas de una manera tan sencilla. Gracias a él me titulé de licenciatura y de maestría [Georgina Yólotl Gallardo, maestría en Demografía, generación 1993-1995].

Siempre dispuesto a apoyar a sus alumnos. Gran pérdida para el CEDUA y para la comunidad demográfica en América Latina [Adriana López, maestría en Demografía, generación 1991-1993].

* El Colegio de México, A.C. Correo: sgiorguli@colmex.mx ORCID: https://orcid. org/0000-0003-4573-9389 
Siempre estará presente en quienes fuimos sus alumnos [Larissa Ibarra, maestría en Estudios Urbanos, generación 2005-2007].

Mi mejor profesor de matemáticas [Cecilia Gayet, maestría en Demografía, generación 1997-1999].

Fue profesor de muchos y muchas demógrafas, un formador incansable. Le debo mi gusto por la demografía. Siempre estará presente en nuestros corazones [Edith Pacheco, maestría en Demografía, generación 1985-1987].

Explicaba con precisión y educaba con amabilidad [EsKarlata Lata, maestría en Estudios Urbanos, generación 2013-2015].

Las descripciones y las experiencias de sus exalumnos son reiteradas y coinciden. En mi caso, fue mi maestro en cinco cursos de Análisis Demográfico. Se combinó la ausencia de profesores del entonces CEDDU por sus licencias sabáticas o por sus cargos de gestión académica, lo que llevó a mi generación a tener tantos cursos con Mina -desde el propedéutico hasta el adicional que pedimos para los temas que nos faltaron-. De ahí que a quienes cursamos la generación de la maestría en Demografía 1993-1995 nos llamaran "las hijas de Mina", a mucha honra. Debo confesar que yo aún conservo mi carpeta con los apuntes que tomé en sus clases.

Hay tres aspectos de su forma de ser profesor que quisiera resaltar:

- La facilidad para transmitir las ideas y los conceptos de las matemáticas y del análisis demográfico.

- La habilidad para comunicarse con alumnos de las más diversas disciplinas: desde antropología e historia hasta ingenierías y matemáticas, pasando por economistas, sociólogos, abogados, actuarios y toda la gama de estudiantes que tuvo en el CEDUA y en el Colmex.

- Su infinita paciencia y disponibilidad para dar atención personalizada a sus alumnos.

De éstos y los varios atributos que mencionan sus estudiantes se deriva esa capacidad para motivarlos -como ayudarlos a perder el miedo a las matemáticas-, al menos temporalmente.

$\mathrm{Su}$ apertura hacia los alumnos explica que fuera un director de tesis preferido por muchos. Más de 160 tesis de licenciatura y casi 30 de maestría dirigidas en sus cuatro décadas como profesor dan testimonio de ello. 
Probablemente el más grande legado de Alejandro Mina quede en las múltiples generaciones de demógrafos y apasionados de la demografía que formó en la UNAM y el Colmex.

\section{Mina el demógrafo: apasionado rigor en el análisis demográfico $y$ extremado cuidado en el uso del dato}

El profesor Mina era un convencido de la relevancia y los aportes de la demografía. Al momento de enseñar análisis demográfico, esto se traducía en:

1. una presentación muy ordenada de las técnicas;

2. su énfasis en entender de dónde provenían éstas y su aversión a usar los paquetes estadísticos si antes no habíamos entendido la lógica del método;

3. su empeño en evaluar la calidad del dato, criticarlo, corregirlo si era posible y conocer los límites del mismo.

Era un convencido de la importancia de que demógrafos y no demógrafos que usan las estimaciones de fecundidad, mortalidad, migración y las proyecciones entendieran a cabalidad los conceptos. Gran comunicador, se abrió para hablar de demografía con diversas comunidades. El ejemplo más reciente es el curso masivo en línea que, con apoyo del Fondo de Población de las Naciones Unidas y la Coordinación de Educación Digital del Colmex, grabó en 2017 y que apenas cerró en su segunda emisión.

Cabe señalar que, dentro de su énfasis y su vocación hacia las estimaciones demográficas, reconocía también -en su visión más amplia del oficio de los demógrafos- la necesidad de integrar interpretaciones que incluyeran tanto las estimaciones desde la demografía formal como desde la sociodemografía.

\section{Mi querido profesor Mina}

¿Qué me enseñó mi profesor Mina? Hay mucho en mi propio estilo de docencia que aspira a ser como el suyo; por ejemplo: la generosidad para compartir lo que sabía y la capacidad para dar seguridad a los alumnos y motivarlos a continuar su formación aun en temas que podían parecerles áridos.

En mi caso en particular, y con esto quiero cerrar, me hizo recuperar esa pasión por las matemáticas que tuve desde muy joven (y que quedó perdida 
un poco cuando decidí estudiar Sociología), me dio herramientas para un pensamiento ordenado en el momento de trabajar con el análisis demográfico y estadístico, y fue un gran apoyo desde que me incorporé como profesora al CEDUA en 2002, y después como directora del Centro y como presidenta de El Colegio de México. Primero mi profesor, después mi colega, mi amigo y un gran ser humano al que admiro.

Cierro haciendo mías las palabras de otros de sus alumnos:

Lo recordaremos siempre como un excelente profesor, pero más como una gran persona. Descanse en paz [Adrián Meza, maestría en Demografía, generación 2014-2016].

Nos va a hacer -ya nos hace- mucha falta [Erick Serna, maestría en Estudios Urbanos, generación 2011-2013].

En nuestras memorias y en nuestros corazones, siempre [Olinca Páez, maestría en Demografía, generación 2010-2012]. 


\section{Palabras de Mauricio Rodríguez Abreu}

Quiero empezar agradeciendo la invitación para estar con todos ustedes esta tarde recordando a Alejandro Mina. Agradezco la oportunidad de compartir estas palabras, así como la satisfacción de conocer a su familia, pues no tenía ese gusto más allá de las historias que él me contaba.

Más que enfocarme en la trayectoria de Alejandro Mina, que ya ha sido comentada, o de la forma particular de impartir docencia que él tenía, trataré de explicar lo que representó para nosotros -particularmente para mí-, los de las promociones más jóvenes, cómo lo veíamos y cómo realmente impactó en nuestra formación. Debo empezar también mencionando que dentro de esas 165 tesis de licenciatura que, según señalaban, Alejandro Mina dirigió, está la mía; de las 30 de maestría, también está otra mía, y las de varias personas que he conocido a lo largo de estos años.

Cuando les comenté a algunas personas que estaría aquí esta tarde, muchas se acercaron, me enviaron correos o mensajes, y me comentaron que, aunque no podrían asistir, seguirían la transmisión desde sus centros de trabajo o desde los estados en los que viven. Apenas ayer platicaba con una egresada de la maestría, quien me pidió de manera particular que mencionara cómo le llamábamos a Alejandro: nuestro profe Mina.

Alejandro Mina fue un gran profesor, pero no sólo nos enseñaba matemáticas o técnicas demográficas, lo hizo de tal manera que buscaba a cada uno enseñarle de la manera que fuera más adecuada. Además, debido a sus años de docencia, sabía en qué parte debía hacer énfasis, en dónde nos "perderíamos" y qué hacer para que todos aprendiéramos y alcanzáramos cierto nivel de conocimiento. Para esto, el profe Mina reconocía que no todos necesitábamos la misma atención en todos los temas. Recuerdo que cuando un compañero actuario de la promoción quiso ir al propedéutico, lo corrió; le dijo "Tú no tienes que estar aquí, esto es para ayudar a quienes necesitan más apoyo". Este tipo de acciones muestran cómo Alejandro siempre se interesaba por ayudar a quienes más lo necesitaban, dejando trabajar de manera independiente a quienes él veía que podían hacerlo.

En mi trayectoria personal Mina jugó un papel central. Recuerdo cuando en los primeros semestres de la licenciatura le comenté a una compañera que estaba estudiando actuaría porque quería ser demógrafo. La respuesta de mi compañera fue: "Debes tomar clase con Alejandro Mina". Así conocí a un gran profesor, quien sería mi director de tesis en dos ocasiones y, además,

* Universidad de las Américas Puebla, Departamento de Actuaría, Física y Matemáticas. Correo: mauricio.rodriguez@udlap.mx ORCID: https://orcid.org/0000-0002-7824-7447

Estudios Demográficos y Urbanos, vol. 34, núm. 3 (102), 2019, pp. 715-729

doi: http://dx.doi.org/10.24201/edu.v34i3.1986 
de quien aprendería un modelo de enseñanza que intento seguir y honrar todos los días. Este semestre estoy dando el curso Análisis Demográfico para Actuarios. En este curso, Alejandro siempre hacía énfasis en las técnicas demográficas tradicionales, recalcaba la necesidad de analizar la calidad del dato, y de suavizar la información antes de usarla. En mi curso, acabo de impartir el método de corrección por un dieciseisavo; para esto me basé en unas notas del curso de Demografía de Alejandro Mina, que aún se pueden encontrar en la Facultad de Ciencias de la UNAM. Siempre, ante la duda, regreso a sus notas.

Además de seguir su modelo de enseñanza, Alejandro Mina fue siempre para mí un gran mentor y un gran amigo. Sé que no estaría donde estoy hoy en día de no haber sido por él. Con Alejandro aprendí economía y demografía; fui su ayudante en la Facultad de Ciencias durante algunos años. Cuando me fui a trabajar al Conapo, en lugar de entrar a la maestría en 2008, respetó mi decisión, aunque él hubiese querido que yo estudiara la maestría. Esa fue tal vez la única ocasión que tuvimos un desacuerdo. También tuve la oportunidad de ser tesista de Alejandro en dos ocasiones, y en cada una me dio la libertad para trabajar a mi ritmo y encontrar la forma de hacer la investigación que funcionaba para mí. También gracias a él fue que vine a El Colegio de México por primera vez, a usar la biblioteca, a reunirme con él para comentar los avances de mi tesis. Fue también quien me invitó por primera vez a ser jurado en un examen profesional, durante el cual recuerdo haber estado muy nervioso, incluso más que el día de mi examen. Cuando regresé a México, tras haber terminado mi doctorado, pasé demasiadas horas en su cubículo, no sólo hablando de temas laborales sino de la vida, de mis planes. Además de la parte académica, él siempre tenía un consejo que dar, nos apoyaba, nos daba "terapia", siempre buscó orientarnos y ayudarnos a encontrar nuestro camino. Incluso, debo reconocer que mi trabajo actual lo obtuve siguiendo sus consejos. Siempre lo voy a recordar, siempre va a estar en mi corazón.

No podríamos entender la demografía en el país y la formación de tantos demógrafos mexicanos sin Alejandro Mina. El profe Mina formó no solamente muchos actuarios, matemáticos y demógrafos en México, sino que siempre se interesó por la parte humana de cada uno. Yo no podría entender mi trayectoria profesional sin el rol que jugó Alejandro Mina. Mi historia es similar a la de muchos aquí presentes, pero sé que Alejandro nos recordaba a cada uno de manera muy especial, y sé que siempre lo recordaremos. 
